\title{
Revista Colombiana de

\section{Experiencia inicial con el retiro de electrodos de estimulación cardiaca mediante una técnica de extracción percutánea mecánica}

\author{
Mauricio Duque ${ }^{a, b, *}$, Juan C. Díaz ${ }^{a, b}$, Jorge E. Marín ${ }^{a, b}$, Julián M. Aristizábal ${ }^{a, b}$, \\ Jorge E. Velásquez ${ }^{a, b}$, Laura Duque ${ }^{c}$ y William Uribe ${ }^{a}$
}

a Cardiología y Electrofisiología, CES cardiología, Clínica CES, Medellín, Antioquia, Colombia

b Cardiología y Electrofisiología, Clínica Las Américas, Medellín, Antioquia, Colombia

c Universidad CES, Medellín, Antioquia, Colombia

Recibido el 13 de octubre de 2014; aceptado el 27 de febrero de 2015

Disponible en Internet el 5 de mayo de 2015

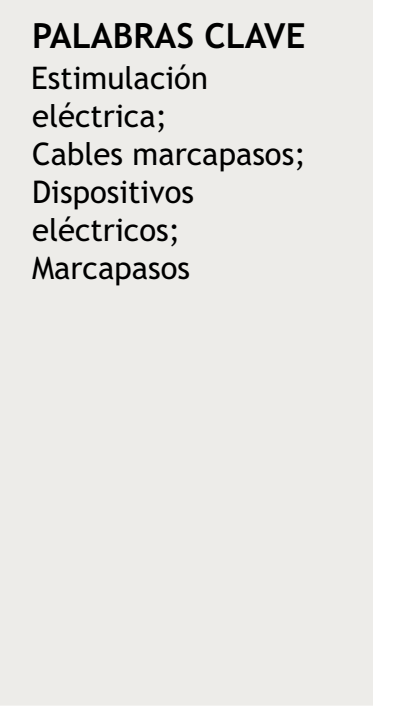

\begin{abstract}
Resumen
Introducción: En Colombia el uso de técnicas de extracción de electrodos de estimulación cardiaca es infrecuente, en parte debido al alto costo de los materiales utilizados para la extracción percutánea y por otra parte por los riesgos percibidos asociados al procedimiento. Esto ha llevado a que muchos electrodos disfuncionantes o infectados sean abandonados o retirados mediante cirugía abierta. Con base en lo anterior se ha desarrollado un programa de extracción de electrodos mediante el uso de vainas mecánicas que pretende evitar la cirugía cardiaca y mantener un perfil de costo beneficio favorable en comparación con las vainas de extracción láser.

Materiales y métodos: Desde noviembre de 2012 hasta septiembre de 2014, aquellos pacientes con indicación para la extracción de electrodos fueron sometidos a dicho procedimiento mediante el uso de vainas mecánicas. De manera prospectiva, en un formulario previamente diseñado se registraron los datos demográficos de los pacientes, las características de los dispositivos, la clase de electrodos y el tipo de complicaciones asociadas al procedimiento. Resultados: Treinta y siete pacientes ( 29 hombres, $78 \%$ ) con un promedio de edad de 67 años (rango 24-91 años) fueron llevados a extracción de electrodos principalmente por complicaciones de tipo infeccioso $(56,7 \%)$. La tasa de éxito fue del $98,4 \%$ con una complicación mayor $(2,7 \%)$ y una complicación menor $(2,7 \%)$. No se registraron muertes.
\end{abstract}

\footnotetext{
* Autor para correspondencia.

Correo electrónico: mauricioduque@une.net.co (M. Duque).
} 
Conclusión: La extracción de electrodos por electrofisiólogos debidamente entrenados permite obtener resultados muy buenos con una baja tasa de complicaciones. El uso de vainas de extracción mecánica es una alternativa viable y de costo razonable en nuestro medio sin necesidad de recurrir a cirugía cardiaca o vainas de extracción láser.

(c) 2014 Sociedad Colombiana de Cardiología y Cirugía Cardiovascular. Publicado por Elsevier España, S.L.U. Este es un artículo Open Access bajo la licencia CC BY-NC-ND (http://creativecommons.org/licenses/by-nc-nd/4.0/).

\section{KEYWORDS}

Electrical stimulation; Pacemaker leads; Electronic devices; Pacemakers
Initial experience with mechanical percutaneous extraction of cardiac pacing leads

\begin{abstract}
Introduction: The extraction of cardiac pacing leads is infrequent in Colombia, partly owing to the high cost of the devices used during the percutaneous procedure and to the widespread perception of its unacceptably high risk. This has led to a large number of malfunctioning or infected leads abandoned or surgically removed. With this in mind, we developed a mechanical lead extraction program aiming to avoid surgical removal of leads while at the same time maintaining reasonable costs in comparison with laser based extraction devices.

Materials and methods: Between November 2012 and September 2014, every patient who required lead extraction according to current guidelines was included. Patient characteristics, lead type, type of cardiac stimulation device, reason for extraction and complications where prospectively registered in a previously designed questionnaire.

Results: A total of 37 patients (29 male, $78 \%$ ) with an average age of 67 years (range 24-91 years) required mechanical lead extraction, most of them infection-related $(56.7 \%)$. Procedural success was $98.4 \%$, with 1 major complication (2.7\%) and 1 minor complication (2.7\%). There were no deaths.

Conclusions: Lead extraction undertaken by well-trained electrophysiologists has excellent results with a low rate of major and minor complications. The use of mechanical lead extraction devices is a viable option with a reasonable cost in our country, avoiding the need for surgical removal of leads or the need of laser devices.

(C) 2014 Sociedad Colombiana de Cardiología y Cirugía Cardiovascular. Published by Elsevier España, S.L.U. This is an open access article under the CC BY-NC-ND license (http://creativecommons.org/licenses/by-nc-nd/4.0/).
\end{abstract}

\section{Introducción}

Los dispositivos de estimulación cardiaca como los marcapasos, los resincronizadores y los cardiodesfibriladores han logrado un impacto significativo, de acuerdo a la patología intervenida, en la calidad de vida y/o en la expectativa de vida de las personas, lo que lleva a un notorio aumento de su uso a nivel mundial. A pesar de que la tasa de complicaciones (incluyendo las infecciones, los desplazamientos de electrodos, y/o la disfunción de estos o de sus componentes) es relativamente baja, el gran número de pacientes que anualmente reciben este tipo de dispositivos implica un volumen considerable de pacientes con complicaciones asociadas. Muchos de estos pacientes van a requerir explante de los dispositivos, y es aquí donde la extracción de electrodos desempeña un papel importante ya que permite disminuir la cantidad de material protésico que se encuentra en el espacio intravascular y en las cámaras cardiacas. Inicialmente, la única opción con la que se contaba para el manejo de estos pacientes era a través de la cirugía abierta, lo que se asocia a una mayor morbilidad y mayores costes para el sistema de salud. Posteriormente, aparecen los dispositivos de extracción mecánica (las vainas dilatadoras, los dispositivos rotatorios, los estiletes para extracción) y de manera más reciente las vainas láser. Los costos de estos dispositivos son elevados, particularmente el de las vainas láser, lo que ha limitado su uso en economías en desarrollo. Esto, sumado al entrenamiento que se requiere y los riesgos inherentes del procedimiento, ha llevado a que el número de personas entrenadas en la extracción de electrodos es menor a la esperada, particularmente en nuestro medio.

En un intento por aplicar los estándares internacionalmente aceptados para la extracción de electrodos, nuestro grupo se ha entrenado en técnicas de extracción mecánica, creando un protocolo para el manejo de estos pacientes durante el procedimiento. Describimos a continuación nuestra experiencia inicial con esta técnica, que busca obtener una mejor solución para los pacientes con dicha indicación y disminuir el número de electrodos abandonados en las cavidades cardiacas y el espacio vascular que podrían ocasionar complicaciones futuras. Así mismo, buscamos evaluar la utilidad de las vainas de extracción mecánica y determinar la verdadera necesidad de usar vainas de extracción láser en nuestro medio.

\section{Selección de los pacientes}

Desde noviembre de 2012, aquellos pacientes con indicación para la extracción de electrodos (independiente del tipo de 
electrodo, sea de estimulación auricular o ventricular, electrodos de alta energía o de seno coronario) de acuerdo al consenso actual $^{1}$, fueron evaluados para determinar si se realizaba o no la extracción percutánea de los electrodos. En aquellos pacientes en quienes el tiempo desde el implante fue mayor a seis meses se consideró el uso de dispositivos de extracción mecánica, mientras que en los pacientes con menos de seis meses desde el implante se consideró innecesario el uso de estos dispositivos ${ }^{2}$. Inicialmente, se seleccionaron aquellos pacientes con complicaciones infecciosas y una vez se tuvo más experiencia con la técnica, se incluyeron pacientes con electrodos disfuncionantes, en quienes hasta el momento ha sido costumbre en nuestro medio el abandono del electrodo y la inserción de uno nuevo. Se excluyeron aquellos pacientes con comorbilidades severas o una expectativa de vida corta, en quienes los riesgos del procedimiento quirúrgico ampliamente superan los beneficios de la extracción del electrodo.

\section{Descripción de la técnica quirúrgica}

Los procedimientos fueron realizados por un equipo multidisciplinario incluyendo el grupo de electrofisiología, anestesiología, cirugía cardiovascular y enfermería con al menos dos operadores entrenados en la técnica de extracción. Dado que en el país no hay hasta el momento un centro de referencia que permita el entrenamiento en técnicas de extracción, varios miembros del grupo se han entrenado en el exterior con grupos especializados en extracción de electrodos. En total, el equipo de extracción está compuesto por siete personas: un electrofisiólogo que actúa como primer operador, un segundo operador entrenado en extracción de electrodos, un anestesiólogo, un cirujano cardiovascular (que en los primeros casos estaba dentro de la sala de procedimientos y actualmente está de disponibilidad inmediata), una enfermera profesional y una auxiliar de enfermería entrenadas en procedimientos de electrofisiología, y una circulante de quirófano. La extracción se realiza en una sala de electrofisiología híbrida y se tiene disponibilidad de si se complica el paciente realizarle la intervención quirúrgica en la misma sala (incluyendo equipos y personal necesario en caso de requerir circulación extracorpórea), debido a la posibilidad de perforación cardiaca o rotura vascular durante la extracción.

Previo al inicio del procedimiento se evalúan el número de electrodos, su ubicación mediante radiografía de tórax posteroanterior y lateral, el uso de tomografía computarizada se reserva para aquellos pacientes en los cuales el curso intravascular de los electrodos no queda claramente definido mediante el uso de radiografía simple. Adicionalmente, se establecen las características de los electrodos (la construcción coradial vs. la construcción coaxial, la fijación activa vs. la fijación pasiva) con el fin de trazar un plan quirúrgico claro.

Con el paciente bajo anestesia general y monitoría continua (que incluye capnografía, presión arterial invasiva, oximetría y uso de parches de desfibrilador) se procede a realizar una disección por planos mediante el uso de electrobisturí. Este permite liberar en corto tiempo el dispositivo y los electrodos, hacer la capsulectomía (utilizando el mismo dispositivo como plano de clivaje) y realizar la disección del

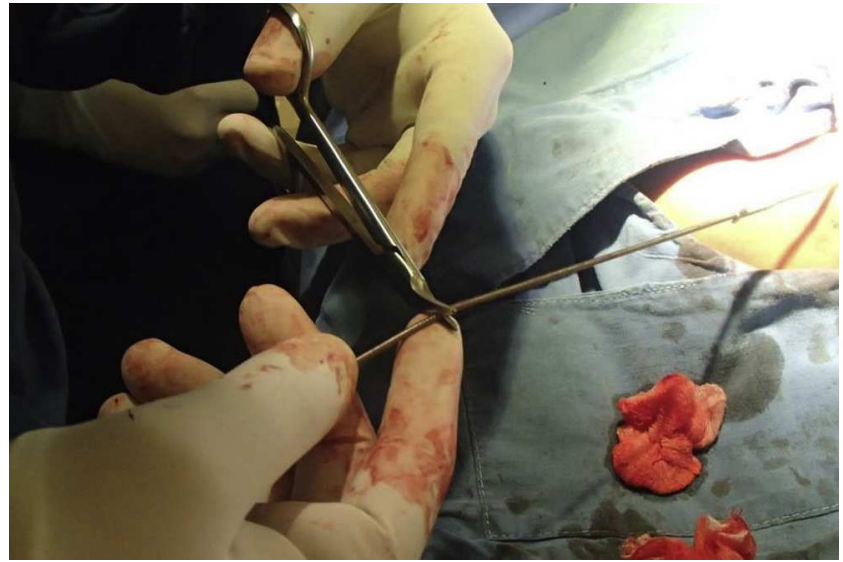

Figura 1 Una vez se ha liberado el trayecto del electrodo en su totalidad, se procede a cortar el extremo conector del electrodo. El corte debe ser limpio, con el fin de preservar la luz interna y facilitar el paso de la guía de fijación interna.

cable hasta su sitio de acceso vascular sin daño a los electrodos. Una vez liberado, se extrae el dispositivo y se procede a liberar el mecanismo de fijación (para los electrodos de fijación activa) y se realiza un intento de extracción mediante tracción simple (definida como la tracción del electrodo sin ningún tipo de elementos adicionales). Si no se logra retirar el electrodo, se realiza una disección hasta el sitio de acceso vascular y posteriormente se corta el extremo conector del electrodo (fig. 1) y a través de la luz interna se introduce la guía de fijación interna (LLD EZ ${ }^{\circledR}$, Spectranetics, Colorado Springs, Colorado) hasta la porción distal del electrodo, una vez se encuentra en este punto se procede a activar su mecanismo de fijación interna (fig. 2). Teniendo ya el electrodo que se encuentra fijo, se introduce una vaina dilatadora

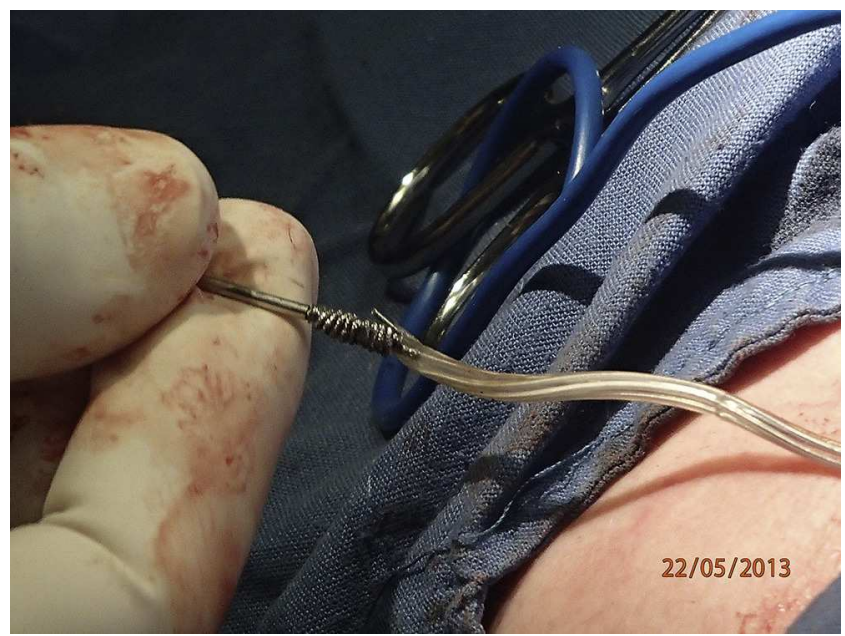

Figura 2 A través de la luz interna se avanza el LLD ${ }^{\circledR}$ vigilando mediante guía fluoroscópica su ubicación dentro del electrodo. Idealmente se debe avanzar hasta la punta del electrodo con el fin de asegurar una tracción que se produzca desde la punta del electrodo, disminuyendo el riesgo de rotura y fragmentación del mismo. Cuando el LLD ha avanzado hasta su posición final, se retrae el dispositivo de fijación, hacer esto antes de tiempo impide la posterior movilización y reacomodación del LLD. 


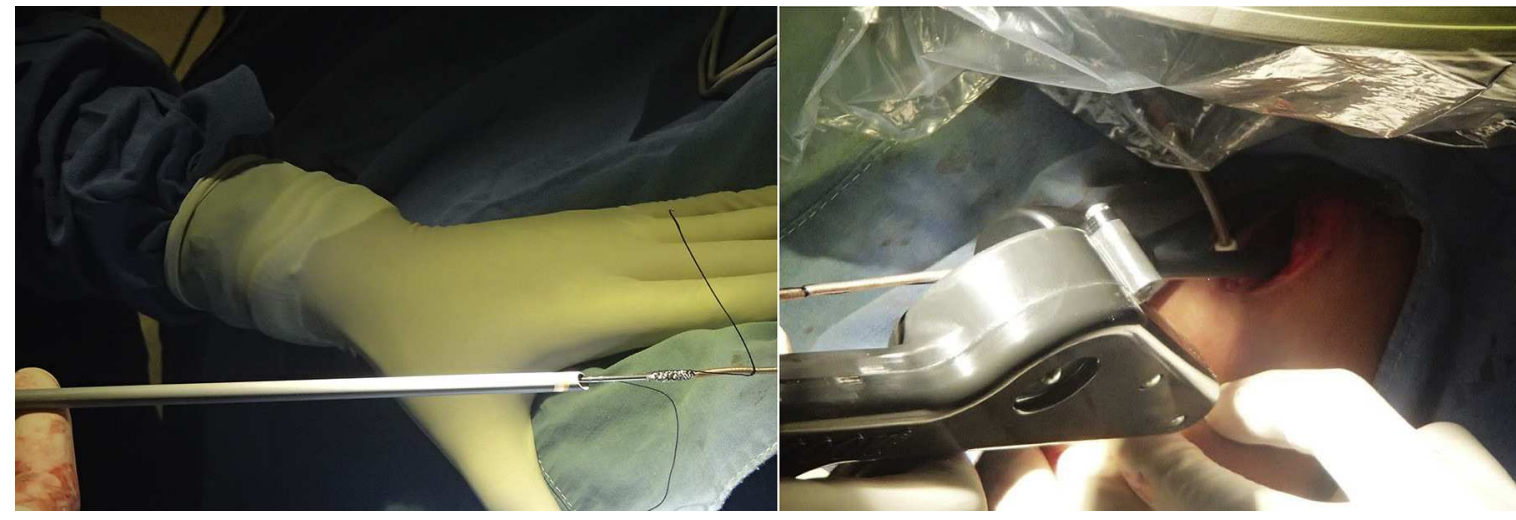

Figura 3 Una vez el electrodo se ha fijado, se procede a introducir la vaina dilatadora (izquierda) o el sistema de extracción Evolution ${ }^{\circledR}$ (derecha). En este punto es fundamental buscar una adecuada alineación de la herramienta de extracción con el vaso, con el fin de facilitar su avance.

(VisiSheath $^{\circledR}$, Spectranetics, Colorado Springs, Colorado, EE. UU.) y se avanza ejerciendo contratracción con la guía de fijación interna hasta lograr la liberación del electrodo. Adicionalmente, contamos con dispositivos de rotación Cook Evolution (Cook Medical, Bloomington, Indiana, EE. UU.) los cuales se utilizan cuando el avance de la vaina dilatadora es difícil debido a la presencia de tejido fibrótico denso alrededor del electrodo (fig. 3). Este dispositivo utiliza un movimiento rotacional de su cabeza para cortar las adherencias y liberar el electrodo del tejido circundante. Este procedimiento se repite con cada uno de los electrodos, una vez extraídos se cierra el sitio de acceso vascular y posteriormente se realiza el cierre de la herida por planos anatómicos usando sutura bioabsorbible.

\section{Resultados}

Entre diciembre de 2012 y septiembre de 2014 un total de 37 pacientes (29 hombres, $78 \%$ ) con un promedio de edad de 67 años (rango 24-91 años) fueron llevados a extracción de electrodos. Las razones que llevaron a la extracción de los dispositivos se muestra en la tabla 1 con ejemplos en la figura 4, siendo la causa más frecuente la disfunción del electrodo $(35,1 \%$ de los casos) seguida por la extrusión del cable o del dispositivo $(24,3 \%)$ y la infección del bolsillo $(16,2 \%)$. El total de causas relacionadas con procesos infecciosos (bacteriemia, endocarditis infecciosa, extrusión del electrodo o el dispositivo, infección del bolsillo), representó el $56,7 \%$ de los casos de extracción. Estos

Tabla 1 Indicaciones para la extracción de los electrodos

\begin{tabular}{lc}
\hline Indicación & No. de pacientes (\%) \\
\hline Disfunción del electrodo & $13(35,1)$ \\
Extrusión del cable o dispositivo & $9(24,3)$ \\
Infección de bolsillo & $6(16,2)$ \\
Endocarditis infecciosa & $4(10,8)$ \\
Bacteriemia por grampositivos & $2(5,4)$ \\
Dolor intratable & $1(2,7)$ \\
Perforación del VD por el electrodo & $1(2,7)$ \\
\hline
\end{tabular}

37 pacientes tuvieron un total de 63 electrodos considerados para la extracción (algunos pacientes tenían electrodos que se buscó preservar), representados por 23 electrodos auriculares, 16 electrodos de alta energía (incluyendo tres electrodos Riata), 18 electrodos de estimulación ventricular derecha y seis electrodos ventriculares izquierdos. Durante uno de los procedimientos se presentó daño a un electrodo de alta energía al intentar retirar un electrodo auricular, este electrodo de alta energía se incluyó en el análisis de tasa de éxito (total: 64 electrodos para dicho análisis). De los cables extraídos, 26 (41\%) fueron retirados mediante tracción simple, incluyendo un electrodo de desfibrilación. La extracción mediante tracción simple fue más exitosa en aquellos pacientes con implantes de menos de dos años que en aquellos pacientes cuyo implante había sido realizado hacía más de dos años.

\section{Complicaciones}

Las complicaciones asociadas tanto intraprocedimiento como periprocedimiento (definido como el período de tiempo en los 30 días siguientes al procedimiento) se clasificaron utilizando el esquema propuesto actualmente ${ }^{1}$.

- Complicación mayor: aquella que pone en riesgo la vida del paciente, que genera una discapacidad física significativa o que requiere intervención quirúrgica para su corrección con el fin de evitar dichos desenlaces.

- Complicación menor: cualquier otro tipo de complicación que no pone en riesgo la vida o la función del paciente o aquellas que pueden ser solucionadas mediante una intervención quirúrgica menor.

En nuestra serie se presentó una complicación mayor ( $2,7 \%$; rotura contenida de la vena innominada y posterior trombosis de la vena subclavia y la yugular interna izquierda la cual fue manejada médicamente ya que la paciente rehusó a ser llevada a cirugía) (fig. 5); y una complicación menor $(2,7 \%$; sangrado por lesión en el sitio de acceso vascular que requirió reparo quirúrgico). Las características de los pacientes en las que se presentaron dichas complicaciones se pueden observar en la tabla 2. 

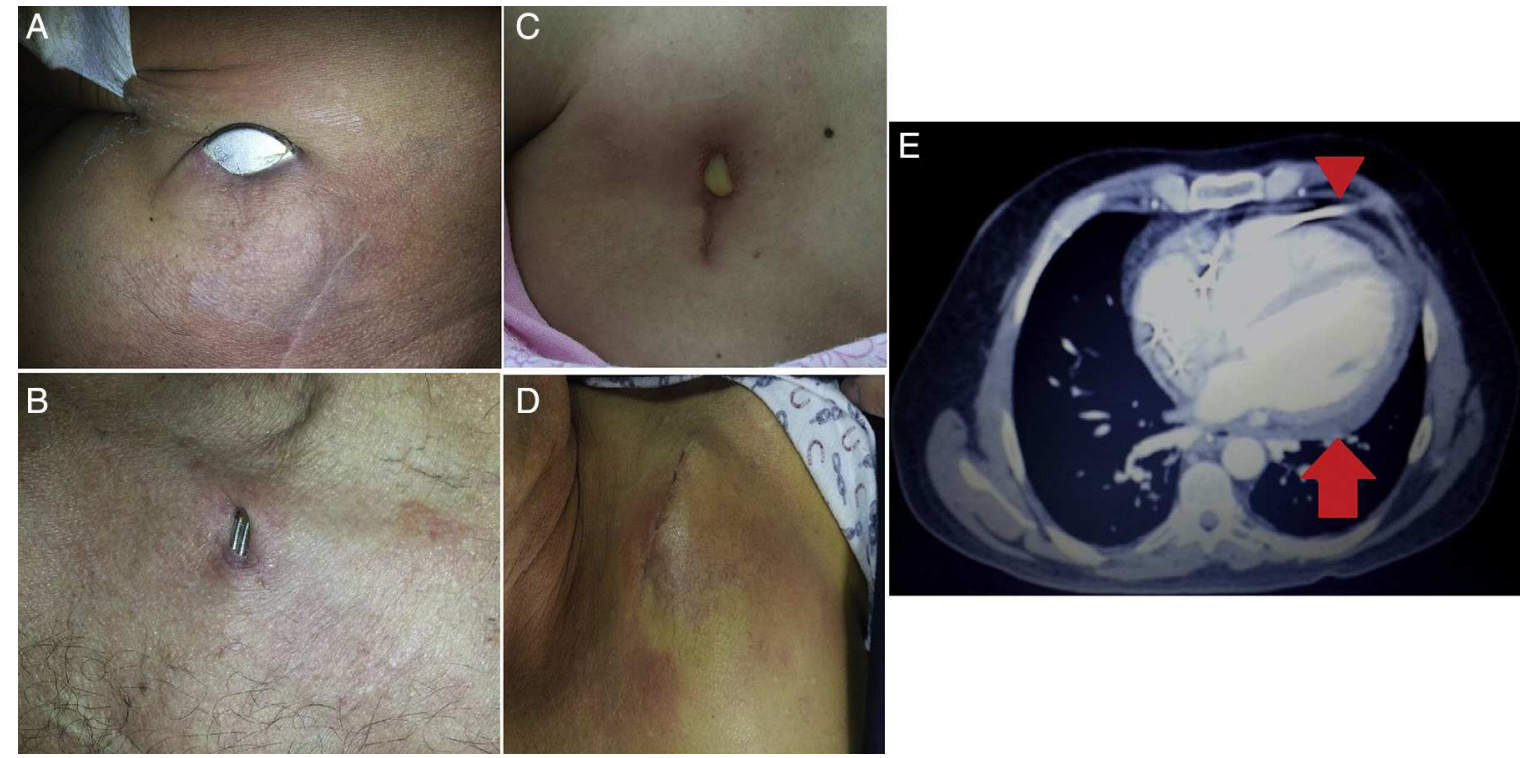

Figura 4 Motivos de extracción de electrodos. Las causas infecciosas fueron las más comunes, ya fuera por extrusión del dispositivo A) o de los electrodos B); o por infección del bolsillo C) y D). Un paciente tuvo perforación del electrodo a través de la pared libre del ventrículo derecho E), cabeza de flecha, lo cual se asoció a dolor precordial y derrame pericárdico E), flecha.

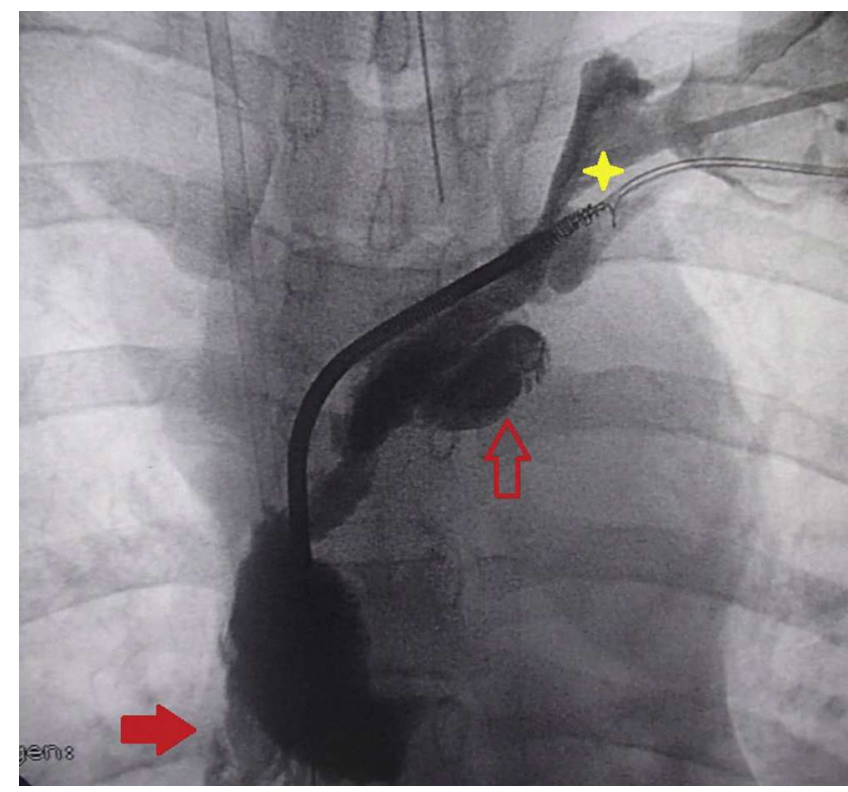

Figura 5 Ruptura contenida de la vena innominada durante un procedimiento de extracción. Se observa daño del electrodo a nivel del coil de alta energía de la vena cava superior (estrella amarilla), ruptura contenida de la vena innominada (flecha hueca) con extravasación del medio de contraste hacia el espacio pericárdico (flecha sólida). El color de esta figura solo puede apreciarse en la versión electrónica del artículo.

\section{Tasa de éxito}

Se lograron extraer mediante la técnica descrita los 62 de los 63 electrodos que habían sido considerados inicialmente para extracción (tasa de éxito de $98,4 \%$ ); no fue posible la extracción de un electrodo de la aurícula derecha el cual había sido cortado cerca de su punto de ingreso al espacio intravascular por debajo de la clavícula derecha lo cual limitó el uso de los dispositivos mecánicos. Incluyendo el electrodo de alta energía que sufrió daño durante la extracción de otro electrodo, se lograron retirar 62 de los 64 electrodos que se intentaron extraer, para una tasa de éxito (definida como el número de electrodos extraídos dividido por el número de electrodos que se intentó extraer) del $96,9 \%$.

\section{Daño y desplazamiento de electrodos adyacentes}

Quince pacientes fueron llevados a extracción de un electrodo (electrodo «target») buscando preservar al menos otro electrodo: cinco con marcapasos bicamerales, tres con cardiodesfibrilador bicameral y seis con cardio-resincronizador y un paciente con un marcapasos unicameral por fibrilación auricular lenta ubicado en el lado derecho que tenía un electrodo adicional en el lado derecho (abandonado) y un electrodo en el lado izquierdo abandonado que sufrió erosión (este último considerado el electrodo target), para un total de 21 electrodos adyacentes. En total, cuatro de esos electrodos adyacentes (19\%) sufrió daños durante el proceso de extracción del electrodo «target» y otros cuatro $(19 \%)$ sufrieron desplazamiento del electrodo (tres de los cuales debieron ser retirados, uno tuvo desplazamiento mínimo que no afectó su funcionamiento). El daño se produjo en tres de los casos durante el proceso de disección (realizada con electrobisturí, produciendo daño del aislamiento) y en otro caso al introducir el dispositivo de rotación Evolution $^{\circledR}$, el cual realizó una torsión sobre el electrodo de alta energía que produjo un desprendimiento de la bobina (asociado a una complicación mayor). Aunque la extracción de este electrodo de alta energía no había sido considerada inicialmente, una vez se documentó el daño se intentó su extracción lo cual no fue posible debido a la aparición 
Tabla 2 Características clínicas de las pacientes que tuvieron complicaciones asociadas al procedimiento. La paciente 15 durante la extracción del electrodo auricular presenta daño del electrodo de alta energía por lo cual también se intentó su retirada

\begin{tabular}{|c|c|c|c|c|c|c|c|c|}
\hline $\begin{array}{l}\text { Paciente } \\
\text { No. }\end{array}$ & Edad & Género & Dispositivo & Diagnóstico & $\begin{array}{l}\text { Indicación } \\
\text { del } \\
\text { explante }\end{array}$ & Complicación & Intervención & Desenlace \\
\hline 2 & 80 & Femenino & $\begin{array}{l}\text { Marcapasos } \\
\text { VDD }\end{array}$ & $\begin{array}{l}\text { Bloqueo } \\
\mathrm{AV} \\
\text { completo }\end{array}$ & $\begin{array}{l}\text { Extrusión } \\
\text { del } \\
\text { dispositivo }\end{array}$ & $\begin{array}{l}\text { Menor: lesión } \\
\text { en el sitio de } \\
\text { acceso vascular }\end{array}$ & $\begin{array}{l}\text { Reparo } \\
\text { quirúr- } \\
\text { gico }\end{array}$ & $\begin{array}{l}\text { Corrección } \\
\text { de la lesión } \\
\text { Implante } \\
\text { posterior de } \\
\text { marcapasos } \\
\text { unicameral }\end{array}$ \\
\hline 15 & 24 & Femenino & $\begin{array}{l}\text { CDI bica- } \\
\text { meral }\end{array}$ & $\begin{array}{l}\text { SQTL } \\
\text { Prevención } \\
\text { secunda- } \\
\text { ria }\end{array}$ & $\begin{array}{l}\text { Disfunción } \\
\text { del } \\
\text { electrodo } \\
\text { auricular } \\
\text { Agotamiento } \\
\text { del } \\
\text { generador }\end{array}$ & $\begin{array}{l}\text { Mayor: rotura } \\
\text { contenida de } \\
\text { vena } \\
\text { innominada; } \\
\text { hemotórax } \\
\text { izquierdo; } \\
\text { trombosis de } \\
\text { subclavia y } \\
\text { yugular interna } \\
\text { izquierda al } \\
\text { intentar } \\
\text { extraer el } \\
\text { electrodo de } \\
\text { alta energía }\end{array}$ & $\begin{array}{l}\text { Tubo de } \\
\text { tórax y } \\
\text { manejo } \\
\text { médico. } \\
\text { Se } \\
\text { planteó } \\
\text { reparo } \\
\text { quirúr- } \\
\text { gico }\end{array}$ & $\begin{array}{l}\text { No aceptó } \\
\text { cirugía. } \\
\text { Pérdida en } \\
\text { el segui- } \\
\text { miento. } \\
\text { No se logró } \\
\text { extracción } \\
\text { del } \\
\text { electrodo } \\
\text { de alta } \\
\text { energía }\end{array}$ \\
\hline
\end{tabular}

de una complicación mayor que obligó a la suspensión del procedimiento (caso No. 15).

\section{Dificultades técnicas}

Las principales dificultades técnicas se presentaron durante el avance de la vaina mecánica en sitios específicos del trayecto del electrodo:

- En el punto de acceso vascular, particularmente cuando el acceso es mediante una punción muy medial que esté en cercanía del ligamento costo clavicular. Este tiende a calcificarse con el tiempo y dificulta la entrada de la vaina. Adicionalmente, el espacio entre clavícula y costilla es menor cuanto más medial se haga la punción, lo cual dificulta aún más la técnica.

- En la curva de la vena braquiocefálica, donde además se debe tener en cuenta la orientación del bisel de la vaina para evitar perforaciones vasculares.

\section{Discusión}

A medida que aumenta el número de dispositivos implantados en nuestro medio, aumenta de manera similar el número de pacientes que requieren retiro de electrodos infectados o disfuncionantes. La tracción simple es insuficiente para lograr el retiro de la mayoría de los electrodos debido al desarrollo de adhesiones fibróticas que los encapsulan, impidiendo su movilización y aumentando el riesgo de complicaciones mecánicas al intentar su retiro. Por esta razón hasta la década de los 90 la conducta más común a nivel mundial era el abandono de los electrodos disfuncionantes, preservando la extracción quirúrgica para aquellos pacientes que tenían infección del dispositivo. Esta conducta ha persistido por más tiempo en nuestro medio, donde la introducción de dispositivos mecánicos para la extracción ha sido lenta y el entrenamiento en técnicas de extracción ha sido insuficiente, en comparación con el auge en el implante de dispositivos de estimulación cardiaca. Si bien podríamos creer que esto es un fenómeno exclusivo de nuestro país, una encuesta reciente en el Reino Unido reportó que un $56 \%$ de los electrofisiólogos entrevistados realizan menos de 20 extracciones anuales ${ }^{3}$. A pesar de que la mayoría de los médicos no respondieron la encuesta (menos del $10 \%$ de los encuestados la respondieron) este estudio permite demostrar cómo en la práctica real la extracción de electrodos por vía percutánea dista de ser una práctica generalizada, aun en países desarrollados.

Nuestros hallazgos permiten establecer que nuestra población es similar a la de otras series, en las que se ha descrito un promedio de edad que oscila entre los 62-67 años con una mayor proporción de hombres que mujeres ${ }^{4-7}$. Las causas que llevaron al retiro de los electrodos son similares a las halladas en otras series, siendo la asociación con infecciones el principal motivo para el retiro de los electrodos ${ }^{4,5}$. En esta situación, el retiro de todo el material protésico (incluidos todos los elementos del dispositivo de estimulación) es indispensable para lograr la resolución del proceso infeccioso. Es frecuente encontrar manejos expectantes en infecciones de dispositivos, particularmente cuando son infecciones de bolsillos, esta conducta solamente lleva a una estancia hospitalaria prolongada, con una alta morbilidad asociada y una tasa de recurrencias muy altas. Se ha 
encontrado que en el $91 \%$ de los pacientes con infección aparentemente localizada en bolsillo los cultivos de los segmentos intravasculares de los electrodos son positivos aun cuando no existan manifestaciones sistémicas, y una conducta expectante en estos casos se asocia a una recurrencia mayor del $90 \%$ a dos años ${ }^{6,8}$. Teniendo en cuenta la tasa de éxito encontrada, la cual es comparable a la encontrada en series mayores e incluso similar a la encontrada en series de extracción de electrodos con vainas láser, creemos que no existen motivos para preferir la extracción quirúrgica a la extracción percutánea en nuestro medio en casos de infecciones del dispositivo, y mucho menos realizar manejo expectante sin retiro del material protésico ${ }^{4,6,7,9}$. Además, la disminución en costos cuando se utiliza la extracción percutánea frente a la extracción quirúrgica o la conducta expectante que lleva a hospitalizaciones y regímenes de antibióticos prolongados, es dramática y debe ser tenida en cuenta a la hora de tomar una decisión.

En nuestra serie se logró la extracción de los electrodos mediante tracción simple en el $41 \%$ de los casos, por encima del $27-29 \%$ descrito por otros grupos ${ }^{2,5,6}$. Este porcentaje tan alto llama la atención, más aún porque se excluyeron aquellos pacientes con electrodos implantados en los seis meses previos, precisamente para disminuir el número de electrodos retirados mediante tracción simple. Es posible que al ser una serie recientemente iniciada, los electrodos extraídos sean más modernos que los que se han extraído en otras series, permitiéndoles soportar mayor tracción sin sufrir daño estructural significativo. Otro grupo ha reportado un éxito aún mayor con la tracción simple de los electrodos, logrando extraer el $84,7 \%$ de ellos a expensas de un $11,9 \%$ de electrodos fracturados que requieren una intervención por vía femoral ${ }^{10}$. Aunque los electrodos que tienen más tiempo desde el implante tienen una mayor posibilidad de tener adherencias, en nuestra serie el electrodo más joven (con apenas 10 meses desde el implante) requirió el uso de dispositivos de extracción mecánica. Es decir que si bien el tiempo desde el implante es una guía para definir si un electrodo puede ser retirado mediante tracción simple, este comportamiento no siempre es predecible y se debe contar con las herramientas y el entrenamiento suficiente para implementar técnicas avanzadas.

Este último punto es fundamental en el momento de evitar complicaciones, ya que la falta de experiencia se refleja en un mayor riesgo periprocedimiento. A pesar de ser un programa joven, nuestra tasa de complicaciones es ligeramente mayor a la descrita en registros de pacientes realizados en centros con gran experiencia pero similar a la de series más pequeñas ${ }^{2,7}$. Este riesgo de complicaciones es mayor en pacientes pequeños (índice de masa corporal $<25 \mathrm{~kg} / \mathrm{m}^{2}$ ), el género femenino, tres o más cables extraídos durante el procedimiento, y una menor experiencia del personal que realiza el procedimiento ${ }^{4,9}$. Las pacientes que presentaron complicaciones en nuestra serie cumplen varios de estos puntos, y siendo un programa en sus fases iniciales aún estamos desarrollando experiencia sobre la marcha. Se sabe por registros internacionales cómo después de los primeros 10-20 casos el riesgo disminuye, con una reducción más evidente al superar los 30 casos $^{1}$. En nuestro caso, la complicación mayor estuvo relacionada con una rotura vascular, que afortunadamente fue contenida ya que la mortalidad para este tipo de lesiones se estima en un $56 \%$ a pesar del manejo quirúrgico siendo particularmente graves las roturas de la vena innominada y de la vena cava superior, y en menor proporción las laceraciones de la aurícula izquierda ${ }^{11}$. Esta curva de aprendizaje es aún más evidente en el manejo de la complicación menor que se tuvo: en retrospectiva, el sangrado que se presentó por el sitio de acceso vascular pudo haber sido controlado durante el mismo procedimiento, con la realización de una jareta con sutura absorbible antes de retirar las vainas mecánicas (maniobra que se incluyó dentro del proceso de extracción después de este evento).

Esta experiencia inicial ha permitido el desarrollo de un protocolo estandarizado que incluye una cuidadosa planeación prequirúrgica y un «plan de vuelo» intraprocedimiento, haciendo la labor de extracción de manera más segura y acortando de manera significativa los tiempos quirúrgicos. Las guías actuales recomiendan un mínimo de 20 procedimientos de extracción al año, creemos que con esta experiencia inicial, que a nuestro parecer ha sido exitosa, hemos logrado vencer el temor que genera este tipo de procedimientos lo que nos permite incluir cada vez más pacientes y casos más complejos. No obstante, somos conscientes de que el desarrollo de estas habilidades requiere una curva de aprendizaje, lo cual ha sido descrito previamente por otros autores a medida que se introducen técnicas de extracción nuevas, y esperamos que con el tiempo los resultados sean tan buenos como los que se reportan en la literatura mundial ${ }^{12,13}$.

Dadas las condiciones económicas de nuestro país es muy probable que la herramienta más importante para la realización de la extracción sean las vainas dilatadoras mecánicas por encima de los sistemas Evolution ${ }^{\circledR}$ y las vainas láser. Nuestra experiencia con el sistema Evolution ${ }^{\circledR}$ no ha sido hasta el momento buena, ya que hemos tenido daño en los electrodos adyacentes y torsión del electrodo «target» con pérdida de la integridad del mismo que dificultan su extracción. Teniendo en cuenta que la tasa de éxito reportada para este sistema es del $82-91,7 \%$ (requiriendo con una mayor frecuencia el uso de técnicas de extracción transfemoral), no encontramos en el momento indicación para continuar con su uso ${ }^{14-17}$.

A pesar de que buena parte de los equipos de extracción se han volcado hacia el uso de vainas láser para las extracciones (particularmente para aquellos casos que de antemano se consideran difíciles), este dispositivo se asocia con una mayor tasa de complicaciones y es mucho más $\operatorname{costoso}^{1,2}$. Recientemente se ha puesto en duda la relación costo beneficio para este método de extracción ya que se calcula en un sobrecosto de hasta el $49 \%$ por electrodo extraído con respecto a una extracción mediante uso de dispositivos mecánicos, con una tasa de éxito en el procedimiento y de complicaciones similares ${ }^{18}$. Es decir, que en nuestro país, las vainas de extracción mecánica son una excelente herramienta de primera línea tal y como sucede en el Reino Unido donde la mayoría de los electrofisiólogos que realizan extracciones utilizan sistemas de extracción mecánicos con vainas mecánicas ${ }^{3}$.

Por último, creemos firmemente que la tendencia de cortar y abandonar los electrodos disfuncionantes debe ser evitada, ya que impide en el futuro lograr extraer dichos electrodos. En nuestra serie, esta fue la razón por la cual no se logró extraer uno de los electrodos. 


\section{Conclusiones}

La extracción de electrodos mediante el uso de dispositivos mecánicos es una alternativa viable y costo efectiva para nuestro medio, en donde aún la disponibilidad de dispositivos de extracción láser es limitada y la relación costo beneficio de estos últimos no es favorable. Si bien existen riesgos de complicaciones significativas asociadas al procedimiento, un adecuado entrenamiento y la planeación meticulosa paso a paso del mismo logran disminuir de manera sustancial el número de complicaciones. El registro de esta experiencia no solo nos permitirá monitorizar nuestro programa de extracción de electrodos sino que además esperamos que motive a otros grupos de nuestro país a realizar avances en este campo.

\section{Responsabilidades éticas}

Protección de personas y animales. Los autores declaran que para esta investigación no se han realizado experimentos en seres humanos ni en animales.

Confidencialidad de los datos. Los autores declaran que en este artículo no aparecen datos de pacientes.

Derecho a la privacidad y consentimiento informado. Los autores declaran que en este artículo no aparecen datos de pacientes.

\section{Conflicto de intereses}

Los autores declaran no tener ningún conflicto de intereses.

\section{Bibliografía}

1. Wilkoff BL, Love CJ, Byrd CL, Bongiorni MG, Carrillo RG, Crossley GH 3rd, et al. Transvenous lead extraction: Heart Rhythm Society expert consensus on facilities, training, indications, and patient management: this document was endorsed by the American Heart Association (AHA). Heart Rhythm. 2009;6(7):1085-104.

2. Marijon E, Boveda S, de Guillebon M, Jacob S, Vahdat O, Barandon $L$, et al. Contributions of advanced techniques to the success and safety of transvenous leads extraction. Pacing Clin Electrophysiol. 2009;32 Suppl 1:S38-41.

3. Sohal M, Williams SE, Arujuna A, Chen Z, Bostock J, Gill JS, et al. The current practice and perception of cardiac implantable electronic device transvenous lead extraction in the UK. Europace. 2013;15(6):865-70.

4. Byrd CL, Wilkoff BL, Love CJ, Sellers TD, Turk KT, Reeves R, et al. Intravascular extraction of problematic or infected permanent pacemaker leads: 1994-1996. U.S. Extraction Database, MED Institute. Pacing Clinical Electrophysiol. 1999;22(9):1348-57.

5. Mazzetti HM. Doce años de extracciones de catéteres endocavitarios de marcapasos y desfibriladores en la Argentina. Rev Arg Cardiol. 2006;74(2):117-22.

6. Kennergren C, Bjurman C, Wiklund R, Gabel J. A single-centre experience of over one thousand lead extractions. Europace. 2009;11(5):612-7.

7. Bongiorni MG, Soldati E, Zucchelli G, di Cori A, Segreti $L$, de Lucia $R$, et al. Transvenous removal of pacing and implantable cardiac defibrillating leads using single sheath mechanical dilatation and multiple venous approaches: high success rate and safety in more than 2000 leads. Eur Heart J. 2008;29(23):2886-93.

8. Klug D, Wallet F, Lacroix D, Marquie C, Kouakam C, Kacet S, et al. Local symptoms at the site of pacemaker implantation indicate latent systemic infection. Heart. 2004;90(8):882-6.

9. Wazni O, Epstein LM, Carrillo RG, Love C, Adler SW, Riggio DW, et al. Lead extraction in the contemporary setting: the LExICon study: an observational retrospective study of consecutive laser lead extractions. J Am Coll Cardiol. 2010;55(6):579-86.

10. De Bie MK, Fouad DA, Borleffs CJ, van Rees JB, Thijssen J, Trines SA, et al. Trans-venous lead removal without the use of extraction sheaths, results of $>250$ removal procedures. Europace. 2012;14(1):112-6.

11. Hauser RG, Katsiyiannis WT, Gornick CC, Almquist AK, Kallinen LM. Deaths and cardiovascular injuries due to device-assisted implantable cardioverter-defibrillator and pacemaker lead extraction. Europace. 2010;12(3):395-401.

12. Bracke FA, Meijer A, van Gelder B. Learning curve characteristics of pacing lead extraction with a laser sheath. Pacing Clinical Electrophysiol. 1998;21 11 Pt 2:2309-13.

13. Ghosh N, Yee R, Klein GJ, Quantz M, Novick RJ, Skanes AC, et al. Laser lead extraction: is there a learning curve? Pacing Clinical Electrophysiol. 2005;28(3):180-4.

14. Hussein AA, Wilkoff BL, Martin DO, Karim S, Kanj M, Callahan T, et al. Initial experience with the evolution mechanical dilator sheath for lead extraction: safety and efficacy. Heart rhythm. 2010;7(7):870-3.

15. Oto A, Aytemir K, Canpolat U, Yorgun H, Sahiner L, Kaya $E B$, et al. Evolution in transvenous extraction of pacemaker and implantable cardioverter defibrillator leads using a mechanical dilator sheath. Pacing Clinical Electrophysiol. 2012;35(7):834-40.

16. Oto A, Aytemir K, Yorgun H, Canpolat U, Kaya EB, Kabakci $G$, et al. Percutaneous extraction of cardiac pacemaker and implantable cardioverter defibrillator leads with evolution mechanical dilator sheath: a single-centre experience. Europace. 2011;13(4):543-7.

17. Mazzone P, Tsiachris D, Marzi A, Ciconte G, Paglino G, Sora N, et al. Advanced techniques for chronic lead extraction: heading from the laser towards the evolution system. Europace. 2013.

18. Starck CT, Rodriguez H, Hurlimann D, Grunenfelder J, Steffel J, Salzberg SP, et al. Transvenous lead extractions: comparison of laser vs. mechanical approach. Europace. 2013;15(11):1636-41. 